\title{
Dez regras práticas para a terapia com corticoides nas doenças inflamatórias em adultos
}

\section{Ten practical rules for corticosteroid therapy in adult's inflammatory diseases}

\author{
Fabricio de Souza Neves ${ }^{1}$
}

1 Professor do Departamento de Clínica Médica, Centro de Ciências da Saúde, Universidade Federal de Santa

Catarina, Campus Reitor João David Ferreira Lima, Florianópolis

Artigo submetido em 22/10/2018

Aceito para publicação em 27/11/2018

Autor para contato: Prof. Fabricio de Souza Neves (fabricio.souza.neves@ufsc.br)

DOI: https://doi.org/10.32963/bcmufsc.v4i11.3339

\section{RESUMO}

Corticosteroides (ou corticoides) são fármacos muito utilizados na prática clínica. Uma de suas principais aplicações é no tratamento de doenças de natureza inflamatória ou autoimune, em que são capazes de aliviar a dor, preservar a função de órgãos vitais e até salvar a vida de pacientes graves. No entanto, seu uso também está associado ao risco de efeitos adversos também graves. Por esses motivos, ter destreza em sua prescrição e manejo dos efeitos adversos é uma competência essencial ao clínico. Para facilitar essa tarefa, este artigo resume conceitos de farmacologia, fisiologia e clínica em relação ao uso de corticoides para o tratamento de doenças inflamatórias e autoimunes em pacientes adultos, na forma de dez regras práticas.

Palavras-chave: Corticoesteroides; Glucocorticoides; Guia; Prednisona; Hidrocortisona; Dexametasona

\begin{abstract}
Corticosteroids are widely used drugs in clinical practice. One of its main applications is in the treatment of inflammatory or autoimmune diseases, in which they can relieve pain, preserve the function of vital organs and even save the lives of critical patients. However, its use is also associated with the risk of serious adverse effects. For these reasons, having skill in prescribing and managing the adverse effects of corticosteroids is an essential clinical competence. In order to facilitate this task, this article summarizes pharmacology, physiology and clinical concepts regarding the use of corticosteroids for the treatment of inflammatory and autoimmune diseases in adult patients, in the form of ten practical rules.
\end{abstract}

Keywords: Corticosteroids; Glucocorticoids; Guideline; Prednisone; Hydrocortisone; Dexamethasone 
Corticosteroide (ou corticoide) significa literalmente "esteroide do córtex". Trata-se do nome dado ao conjunto de compostos bioquímicos de estrutura esteroide produzidos na camada cortical da glândula adrenal que possuem funções hormonais (e seus derivados sintéticos). Estas substâncias têm duas principais atividades: 1) sua ação de regulação da resposta inflamatória e imunológica, também relacionada à ação sobre o metabolismo de carboidratos, que estimula a gliconeogênese e aumenta a concentração sérica de glicose (por isso denominada de ação "glicocorticoide"); 2) sua ação sobre o equilíbrio eletrolítico ou "mineral" (por isso denominada ação mineralocorticoide) que resulta em retenção de sódio, levando ao aumento do volume extracelular, do volume intravascular e da pressão arterial. São hormônios de resposta ao estresse: garantir o volume de fluido circulante através da retenção ávida de sódio, aumentar a disponibilidade de glicose como substrato energético e diminuir a atividade da resposta inflamatória (sentindo menos dor) são ações coordenadas de modo a capacitar 0 organismo a reações de luta pela sobrevivência, em situações de ameaça. ${ }^{1}$

O efeito anti-inflamatório e imunossupressor dos corticoides é a principal razão de sua utilização na prática clínica em doses supra-fisiológicas (seu uso em doses fisiológicas é empregado no tratamento da insuficiência adrenal). Em 1948 foi realizado o primeiro teste clínico com um corticoide: a injeção de 100 mg do composto denominado "cortisona" em pacientes com artrite reumatoide resultou em dramática melhora da doença, até então intratável, fato que abriu caminho para a produção dos diferentes corticoides sintéticos hoje disponíveis e sua utilização em vários distúrbios de natureza inflamatória e autoimune. ${ }^{2}$

Os corticoides são ferramentas (ou armas) clínicas de grande poder. Seu bom uso resulta em alívio da dor, melhora da função de órgãos comprometidos por doenças inflamatórias e, em casos extremos, livra os pacientes da própria morte. Por outro lado, seu uso inadequado causa efeitos adversos às vezes graves e também ameaçadores à vida dos pacientes. Aprender a usar estas ferramentas (ou armas) com maestria é uma necessidade para a boa prática da medicina. Com 0 propósito de facilitar essa tarefa, este artigo apresentará de forma resumida conceitos essenciais de fisiologia, farmacologia e clínica na forma de dez "regras práticas" para a terapia com corticoides.

Nossas regras práticas estão baseadas nos seguintes princípios: 1) existem diferentes corticoides disponíveis, com diferentes potências glicocorticoide e mineralocorticoide; 2) a ação anti-inflamatória e imunossupressora obtida com os corticoides pode ser regulada pela dose empregada e pelo tempo de uso; 3) por serem análogos dos hormônios naturais, os corticoides podem causar supressão da função adrenal, entre outros efeitos adversos, que devem ser adequadamente prevenidos ou manejados.

As regras a seguir tem aplicabilidade para a terapia de condições inflamatórias ou autoimunes em adultos. Não estamos nos referindo à terapia de reposição adrenal nem à terapia em crianças, condições em que a precisão necessária à definição das doses é diferente das situações tratadas neste artigo.

\section{Regra 1: As apresentações mais comuns dos corticoides disponíveis no Brasil são aproximadamente equivalentes em potência glicocorticoide.}

Um comprimido de $20 \mathrm{mg}$ de prednisona se aproxima à potência glicocorticoide de uma ampola de $100 \mathrm{mg}$ de hidrocortisona, a uma ampola (ou um comprimido) de $4 \mathrm{mg}$ de dexametasona, a um comprimido (ou a uma ampola) com $2 \mathrm{mg}$ de betametasona e a um comprimido de $30 \mathrm{mg}$ de deflazacorte; já um comprimido de $5 \mathrm{mg}$ de prednisona se aproxima à potência glicocorticoide de um comprimido de 0,5 $\mathrm{mg}$ de betametasona ou de dexametasona e a um comprimido de $6 \mathrm{mg}$ de deflazacorte.

Essa regra permite um rápido cálculo mental para as doses de corticoides pela sua potência anti-inflamatória e imunossupressora, útil se for necessária uma mudança de prescrição entre estes compostos. Exemplo: se um paciente está em uso de $40 \mathrm{mg}$ de prednisona por dia (dois comprimidos de $20 \mathrm{mg}$ ) e por alguma razão necessita mudar para a via intravenosa, sua terapia glicocorticoide pode ser mantida com o uso de $200 \mathrm{mg}$ de hidrocortisona (duas ampolas de $100 \mathrm{mg}$ ) por dia.

Trata-se de um arredondamento: as doses equivalentes em potência glicocorticoide a $20 \mathrm{mg}$ de prednisona são, na verdade: $80 \mathrm{mg}$ de hidrocortisona (a regra erra em 20\%), 3 mg de dexametasona (a regra erra em 25\%) e 2,4 mg de betametasona (erro de 20\%). Já o comprimido de deflazacorte de $30 \mathrm{mg}$ realmente se equivale a $20 \mathrm{mg}$ de prednisona em potência glicocorticoide. ${ }^{3}$ Para a maioria das situações no tratamento de doenças inflamatórias, essa margem de imprecisão não impede a realização de uma prescrição inicial adequada. Deve-se observar que a metilprednisolona é uma exceção à esta regra, que será abordada na Regra 3.

Regra 2: A hidrocortisona é o corticoide disponível com
maior potência mineralocorticoide. Prednisona e
metilprednisolona possuem efeito mineralocorticoide um
pouco menor, e os demais não têm efeito
mineralocortcoide significativo. Bol Curso Med UFSC 2018; 4(10) 
Tomando-a como referência (100\%), os demais corticoides têm as seguintes potências mineralocorticoides: prednisona $80 \%$; metilprednisolona $50 \%$; já dexametasona, betametasona e deflazacorte não tem potência mineralocorticoide significativa.

Portanto, no que se refere a potência mineralocorticoide, os produtos disponíveis muitas vezes não podem ser intercambiados entre si. Exemplo: um paciente com possível insuficiência adrenal (um usuário crônico de prednisona) entra em choque séptico. A melhor escolha para manutenção de sua corticoterapia por via intravenosa seria utilizar doses equivalentes (ou até mais elevadas) em hidrocortisona ou em metilprednisolona. Caso se optasse por usar dexametasona ou betametasona haveria o risco de não se conseguir manter volume extracelular adequado para obter volume circulante efetivo, devido à ausência de potência mineralocorticoide destes compostos. Por outro lado, o uso crônico de deflazacorte, betametasona ou dexametasona provavelmente acarreta menos efeitos adversos do ponto de vista circulatório, embora estudos comparativos que confirmem essa impressão não existam.

\section{Regra 3: A metilprednisolona é o equivalente intravenoso da prednisona.}

Essa regra define que o tratamento com uma determinada dose de prednisona oral pode ser convertido para 0 tratamento com metilprednisolona intravenosa na mesma dose, e vice-versa, mantendo as mesmas potências glicocorticoide e mineralocorticoide. Exemplo: o paciente em uso de prednisona $60 \mathrm{mg}$ ao dia pode passar a receber $60 \mathrm{mg}$ de metilprednisolona intravenosa por dia, em princípio mantendo-se efeitos similares.

Trata-se de mais um arredondamento: a dose equivalente em potência glicocorticoide a $20 \mathrm{mg}$ de prednisona é, na verdade, de $16 \mathrm{mg}$ de metilprednisolona (a regra erra em 20\%). Já a potência mineralocorticoide da metiprednisolona é, na verdade, cerca de $30 \%$ inferior à da prednisona.

\section{Regra 4: A prednisolona oral é equivalente em potências à prednisona.}

Essa regra define que o tratamento com prednisona pode ser convertido, com as mesmas potências glicocorticoide e mineralocorticoide, em prednisolona com as mesmas doses. A regra é útil no Brasil quando se necessita usar baixas doses de corticoide: pode-se prescrever a prednisolona em gotas, que na apresentação comum de $11 \mathrm{mg} / \mathrm{ml}$ oferece cerca de $0,5 \mathrm{mg}$ por gota (no Brasil não há apresentação de prednisona em doses menores que $5 \mathrm{mg}$ por comprimido).
Regra 5. Os efeitos imunossupressores dos corticoides começam a aparecer clinicamente a partir da dose acumulada equivalente a $600 \mathrm{mg}$ de prednisona, e tornam-se mais intensos progressivamente.

As infecções tornam-se mais frequentes nos pacientes tratados com corticoides, em comparação a placebo, a partir da dose acumulada de $700 \mathrm{mg}$ de prednisona, de onde se pode deduzir que a partir deste valor os pacientes tornam-se clinicamente imunossuprimidos. Obter essa imunossupressão é, geralmente, a meta necessária para se tratar doenças autoimunes. ${ }^{4,5}$

Nossa regra é, novamente, um arredondamento para a clínica prática, pois a dose de $600 \mathrm{mg}$ é múltipla da apresentação comum de prednisona $20 \mathrm{mg}$. De acordo com a regra, quando se prescreve a dose comum de $60 \mathrm{mg}$ ao dia de prednisona, a imunossupressão será alcançada após dez dias (600 mg de dose acumulada). Com a dose de $20 \mathrm{mg}$ ao dia de prednisona, após trinta dias. Quando se usa $5 \mathrm{mg}$ ao dia de prednisona, a imunossupressão só aparecerá após 120 dias. $\mathrm{E}$ quando se faz a pulsoterapia com metilprednisolona intravenosa na dose de $1 \mathrm{~g}$, a imunossupressão é obtida já no primeiro dia. Basicamente, esta é a principal diferença entre o que se costuma denominar na prática de "dose baixa" (5 $\mathrm{mg} / \mathrm{dia}$ ), "dose média" (20 mg/dia), "dose alta" (60 mg/dia) e "pulsoterapia" de corticoides: o tempo que tais doses levam para produzir imunossupressão (meses, semanas, dias ou de imediato, respectivamente).

Regra 6. Os efeitos anti-inflamatórios dos corticoides começam a aparecer clinicamente a partir da dose acumulada de $\mathbf{2 0} \mathrm{mg}$ de prednisona.

Novamente como arredondamento, nossa regra define que a partir da dose acumulada de $20 \mathrm{mg}$ de prednisona (um comprimido) começam a ser sentidos seus efeitos antiinflamatórios. Logo, mesmo antes de se obter ação imunossupressora, em doses menores que aquelas citadas na Regra 5, os corticoides causam efeitos anti-inflamatórios, em geral úteis para o rápido controle de exacerbação aguda de alguma doença inflamatória.

Seis horas após se usar doses de $30 \mathrm{mg}$ a $40 \mathrm{mg}$ de prednisona, verificam-se efeitos de diminuição no processo inflamatório das vias aéreas no tratamento da asma. ${ }^{6}$ Do mesmo modo, o uso de prednisona na dose de $35 \mathrm{mg}$ faz iniciar melhora significativa da inflamação na gota aguda a partir de cerca de 6 horas da administração. ${ }^{7}$ Doses menores implicam em mais tempo para promover melhora de sintomas e sinais inflamatórios. 0 uso de 7,5 mg por dia de prednisona promove melhora significativa de sintomas na artrite reumatoide apenas após cinco dias. ${ }^{7}$ Nossa regra é, portanto, 
um arredondamento prático baseado nestes dados empíricos.

Regra 7. Para se obter ação imunossupressora, uma dose única diária é suficiente, preferencialmente pela manhã; já para uma melhor ação anti-inflamatória aguda deve-se observar uma posologia compatível com a meia-vida do corticoide empregado.

A hidrocortisona tem meia vida curta e para se manter níveis séricos da substância deve ser usada de 6/6h ou de 8/8h; a prednisona, a prednisolona e a metilprednisolona tem meia vida intermediária e podem ser usados de $8 / 8 \mathrm{~h}$ ou de 12/12h; a dexametasona e a betametasona tem meia vida longa e podem ser usados de 24/24h.

Os efeitos imunossupressores são provavelmente mais devidos à ação dos corticoides em modificar (inibindo) a transcrição de genes associados à resposta imune, e por isso essa ação independe da presença do corticoide disponível no plasma ou nos tecidos, desde que ele já tenha penetrado nos núcleos celulares. Embora muitos dos efeitos antiinflamatórios também ocorram por este mecanismo, ao menos parte da ação anti-inflamatória é independente da regulação da transcrição gênica e acontece em nível citoplasmático, da membrana celular ou extracelular, pois ocorre em pouco tempo (antes do tempo necessário à transcrição gênica). ${ }^{8}$ Por isso, uma ação anti-inflamatória mais sustentada ao longo das 24 horas pode ser obtida de forma mais eficiente se a administração do corticoide assegurar níveis plasmáticos e teciduais ideais ao longo de todo o período de tratamento. ${ }^{7}$

Por esta regra entende-se que uma crise aguda de gota, cujo tratamento durará cerca de cinco dias, pode ser melhor tratada com o uso de prednisona $20 \mathrm{mg}$ de $8 \mathrm{em} 8$ horas do que com uma dose única diária de $60 \mathrm{mg}$. Por outro lado, quando se usa corticoide cronicamente com a intenção de se obter imunossupressão (por exemplo, uma paciente com nefrite lúpica em uso de prednisona $60 \mathrm{mg}$ ao dia), usar uma dose única matinal promoverá o efeito terapêutico desejado de imunossupressão (após dez dias, de acordo com a Regra 5) com o benefício de causar menos supressão da função da glândula adrenal, pois haverá períodos do dia sem nível sérico de cortisol exógeno.

Regra 8. Qualquer dose de corticoide usada diariamente por mais de uma semana pode causar supressão da função do córtex da adrenal e após esse período a terapia não pode ser suspensa abruptamente.

Antes de uma semana de tratamento, a terapia com corticoide pode ser suspensa abruptamente com segurança. Após este período, a chance de atrofia com supressão da função do córtex adrenal começa a existir. A produção fisológica de cortisol pela adrenal em uma pessoa adulta pode variar entre cerca de $2 \mathrm{mg}$ a $7 \mathrm{mg}$ de prednisona por dia, em doses equivalentes. Por este motivo, para doses acima de $10 \mathrm{mg} / \mathrm{dia}$ de prednisona, o principal determinante da dose a ser usada é o controle clínico da doença autoimune ou inflamatória de base, e variações abruptas de dose podem ser feitas. Mas ao se trabalhar com doses abaixo de $10 \mathrm{mg} / \mathrm{dia}$ de prednisona, passa a existir o risco de insuficiência adrenal em situações de estresse, e a redução gradual da dose abaixo deste limite é necessária para permitir tempo para a recuperação da produção endógena de cortisol. A recomendação é que se reduza a dose de prednisona (ou prednisolona) na velocidade de $10 \%$ da dose usada a cada semana, ou seja, $1 \mathrm{mg}$ de prednisona por semana. ${ }^{9}$

Esta redução gradual pode ser feita com 0 uso da apresentação de prednisolona $11 \mathrm{mg} / \mathrm{ml}$ em gotas, em que duas gotas equivalem a $1 \mathrm{mg}$ de prednisolona: 20 gotas/dia $(10 \mathrm{mg})$ por uma semana; 18 gotas/dia $(9 \mathrm{mg})$ por uma semana; 16 gotas/dia (8 mg) por uma semana e assim sucessivamente. Um arredondamento prático corresponde à redução de meio comprimido de prednisona $5 \mathrm{mg}$ a cada duas semanas: $10 \mathrm{mg} / \mathrm{dia}$ por duas semanas; $7,5 \mathrm{mg}$ por duas semanas; $5 \mathrm{mg} / \mathrm{dia}$ por duas semanas; $2,5 \mathrm{mg} / \mathrm{dia}$ por duas semanas; $2,5 \mathrm{mg}$ em dias alternados por mais duas semanas.

\section{Regra 9. Os efeitos adversos da corticoterapia devem ser previstos, prevenidos e manejados a partir da dose imunossupressora.}

Em todos os pacientes em que a dose imunossupressora de corticoides seja alcançada (600 mg de prednisona em dose acumulada, Regra 5) o risco de efeitos adversos torna-se significativo e se recomenda: a) Pelos riscos à saúde óssea: otimizar a ingesta de cálcio e vitamina $\mathrm{D}$ e avaliar o risco de fratura (clinicamente e por densitometria óssea), que pode indicar a necessidade de terapia medicamentosa da osteoporose, e radiografar os locais em que surgirem queixas de dor óssea, a procura de fraturas ou necrose óssea; ${ }^{10}$ b) Pela retenção de sódio, edema e hipertensão: reduzir a ingesta de sódio e controlar a pressão arterial, com a introdução de anti-hipertensivos quando indicado, particularmente de diuréticos nas situações de edema; c) Pelos efeitos no metabolismo dos carboidratos e lipídios: reduzir o aporte calórico, controlar o peso e os níveis glicêmicos e de colesterol, tratando-os quando necessário; d) Pelo risco de infecções oportunistas: testar a presença de estrongiloidíase ou tratar empiricamente com ivermetina por dois dias no início da corticoterapia; 11 atualizar o calendário vacinal do paciente, com destaque para as vacinas contra pneumonia e contra influenza sazonal, sendo que as vacinas 
baseadas em microorganismos vivos não devem ser aplicadas se já se produziu a imunossupressão pela corticoterapia; ${ }^{12}$ embora não se justifique para todos os pacientes imunossuprimidos por corticoide, pode-se considerar a profilaxia contra pneumocistose e 0 tratamento da tuberculose latente em casos de maior risco; ${ }^{13}$ e) Pelo risco de catarata: indicar avaliação oftalmológica; f) Pelos efeitos neuropsiquiátricos: comunicar paciente e familiares da possibilidade de alterações do sono e do humor a fim de permitir diagnóstico e tratamento precoces (insônia e hiperatividade são comuns, mas situações mais graves de mania e psicose podem surgir, sendo mais prováveis em pacientes com antecedentes destes distúrbios); g) Pelos efeitos dematológicos: comunicar o paciente das alterações dermatológicas e estéticas (acne, hirsutismo, face cushingoide, obesidade centrípeta, estrias cutâneas), que em sua maior parte serão reversíveis e podem ser minimizadas com terapias locais.

\section{Regra 10. Deve-se buscar usar a menor dose necessária de corticoides para se controlar a doença que se deseja manejar, pelo menor tempo possível.}

A recomendação de se buscar rapidamente a menor dose necessária de corticoterapia em benefício do paciente é a mais clássica regra deste decálogo e foi enunciada pelo próprio inventor da corticoterapia: o Dr. Phillip Hench a proferiu em seu discurso no recebimento do prêmio Nobel, em 1950, ao relatar as limitações e os cuidados que deveriam ser seguidos no uso de sua descoberta. ${ }^{14}$

Hoje, passados exatos 70 anos do primeiro uso da "cortisona", os corticoides ainda são indispensáveis à prática clínica e as orientações para seu melhor uso são conhecimento útil a todo clínico.

\section{Referências}

1. Shaikh S, Verma H, Yadav N, Jauhari M, Bullangowda J. Applications of steroid in clinical practice: a review. ISRN Anestesiology 2012; 1-11. DOI 10.5402/2012/985495

2. Glyn J. The discovery and early use of cortisone. JR Soc Med 1998; 91: 513-517.

3. Parente L. Deflazacort: therapeutic index, relative potency and equivalent doses versus other corticosteroids. BMC Pharmacol Toxicol 2017; 18: 1-8. DOI 10.1186/s40360-016-0111-8

4. Stuck AE, Minder CE, Frey FJ. Risk of infectious complications in patients taking glucocorticosteroids. Rev Infect Dis 1989; 6: $954-963$.

5. Dixon WG, Kezouh A, Bernatsky S, Suissa S. The influence of systemic glucocorticoid therapy upon the risk of non-serious infection in older patients with rheumatoid arthritis: a nested case-control study. Ann Rheum Dis 2011; 70: 656-960.

6. McFadden ER Jr. Dosages of corticosteroids in asthma. Am Rev Respir Dis 1993; 147: 1306-1310.

7. Janssens HJ, Janssen M, van de Lisdonk EH, van Riel PL, van Weel C. Use of oral prednisolone or naproxen for the treatment of gout arthritis: a double-blind, randomised equivalence trial. Lancet 2008; 371: 1854-1860.

8. Coutinho AE, Chapman KE. The anti-inflammatory and immunosuppressive effects of glucocorticoids, recent developments and mechanistic insights. Mol Cell Endocrinol 2011; 335: 2-13.

9. Alves C, Robazzi TCM, Mendonça M. Withdrawal from glucocorticosteroid therapy: clinical practice recommendations. J Pediatr (Rio J) 2008; 84: 192-202.

10. Bukley L, Guyatt G, Fink HA et al. 2017 American College of Rheumatology guideline for the prevention and treatment of glucocorticoidinduced osteoporosis. Arthritis Rheum 2017; 69: 1521-1537

11. Santiago M, Leitão B. Prevention of strongyloides hyperinfection syndrome: A rheumatological point of view. Eur J Intern Med 2009; 20: $744-748$.

12. Luz KR, Souza DCC, Ciconelli RM. Vaccination for immunocompromised patients and patients with autoimmune rheumatic diseases. Rev Bras Reumatol 2007; 47: 106-113.

13. Youssef J, Novosad SA, Winthrop KL. Infection risk and safety of corticosteroid use. Rheum Dis Clin North Am 2016; 42: 157-176.

14. Hench PS. Nobel Lecture: The reversibility of certain rheumatic and non-rheumatic conditions by the use of cortisone or of the pituitary adrenocorticotropic hormone. Nobelprize.org. Nobel Media AB 2014. Web. 18 Jul 2018. http://www.nobelprize.org/nobel_prizes/medicine/laureates/1950/hench-lecture.html 


\section{Sobre o "Boletim do Curso de Medicina da UFSC"}

\section{Submissões para publicação:}

Preferencialmente online através do sistema Open Journals System. Acesso em: http://ojs.sites.ufsc.br/index.php/medicina

Crie seu login e senha para acesso à revista através da página de cadastro, clicando em "Sobre" e em "Submissões"

\section{$\underline{\text { Diretrizes para Autores }}$}

Trabalhos enviados para publicação devem ser montados em arquivo .doc ou .docx, em língua portuguesa, com a seguinte estrutura:

Página de rosto: indicando a sessão em que pretende publicar o trabalho (veja detalhes das sessões em "Políticas de Sessão"), título, identificação dos autores em ordem de citação (nome completo, setor de trabalho, titulação e email de contato), endereço para correspondência do autor responsável pelos contatos.

A declaração de conflitos de interesse dos autores deve aparecer na folha de rosto e será publicada junto ao trabalho. Incluir fontes de apoio financeiro ao trabalho e citar se os autores possuem vinculação a empresas/coletivos/partidos que tenham interesse na divulgação das informações do trabalho.

Deve ser anexado um documento .pdf contendo o seguinte texto: "Declaração de concordância - os autores abaixo assinados declaram que concordam com a publicação do trabalho intitulado (escrever o título do trabalho) em "Boletim do Curso de Medicina da UFSC" e que aceitam as normas de publicação neste periódico. Os autores declaram os seguintes conflitos de interesse: (declarar ligação a empresas/coletivos/partidos/movimentos com interesse na divulgação das informações do trabalho). Cidade, data - Nome completo, e-mail e assinatura de todos os autores"

Segunda página (esta, em português e em inglês): Resumo com até 250 palavras (estruturado da mesma forma que 0 artigo), seguido de três a seis palavras-chave, separadas por ponto-e-vírgula, para indexação (baseadas no DECs - descritores em ciências da saúde)

Em seguida, o artigo (com o máximo de 3000 palavras). Se artigo original, estruturado em seções (Introdução, Objetivos, Método, Resultados, Discussão e Conclusões). Artigos de revisão narrativa podem ter estrutura Introdução, Método, Revisão e Conclusões. As sessões "Artigos", "o que o residente lê" e "Cartas" tem estrutura livre e não exigem resumo.

Trabalhos envolvendo pesquisa com seres humanos ou animais devem mencionar a aprovação no respectivo Comitê de Ética em Pesquisa. Ensaios clínicos devem mencionar o registro em órgão competente.

O artigo deve ser escrito em fonte Tymes New Roman, tamanho 12, espaço simples. As referências devem seguir a norma de Vancouver, conforme divulgadas nas orientações para o Trabalho de Conclusão de Curso da Medicina (http://www.medicina.ufsc.br/files/2011/11/NORMAS-TCC-MEDUFSC-2011.pdf) e nas orientações de normatização de trabalhos acadêmicos da Biblioteca Universitária (http://portal.bu.ufsc.br/normalizacao/), sendo numeradas ao longo do texto em algarismos arábicos sobrescritos.

Figuras e tabelas devem seguir as mesmas normas do TCC da medicina (http://www.medicina.ufsc.br/files/2011/11/NORMAS-TCC-MEDUFSC-2011.pdf). Tabelas podem ser incorporadas no mesmo arquivo do texto; figuras podem ser incorporadas no texto, mas também devem ser encaminhadas como "arquivo suplementar". 